\title{
Türkiye Faunası İçin İki Yeni Neophyllobius Akar (Acari: Prostigmata: Camerobiidae) Kaydı
}

\section{Mustafa AKYOL ${ }^{1}$ iD \\ ${ }^{1}$ Manisa Celal Bayar Üniversitesi, Fen Edebiyat Fakültesi, Biyoloji Bölümü, Manisa \\ 凶:makyol77@gmail.com}

\section{ÖZET}

Bu çalışmada, Kıyı Ege'den tespit edilen Türkiye faunası için yeni kayit olarak tanımlanan Neophyllobius lamimani McGregor, 1950 ve Neophyllobius parthenocissus Bolland, 1991 türleri yeniden tanımlanmıştır. Pinus brutia ve Thymus sp. altı toprak ve döküntü örneğinden alınan akarlar, Berlese düzeneğiyle ayrıştırılarak modifiye Hoyer ortamında preparatı hazırlanmıştır. Daha sonra örneklerin çizim tüpü ataşmanlı mikroskop kullanılarak çizimleri ve ölçümleri yapılmıştır. Çalışmada türlerin Dünya'da yayılış gösterdiği yerlerdeki örnekleri ile karşılaştırılarak benzerlik ve farklılıkları tartışlmıştır.

\section{DOI:10.18016/ksudobil.304398}

\author{
Makale Tarihçesi \\ Geliş : 06.04.2017 \\ Kabul : 27.04.2017
}

Anahtar Kelimeler
Acari,
Camerobiidae,
Neophyllobius,
Yeni kayit,
Türkiye

\section{Araştırma Makalesi}

\section{Two New Records of Neophyllobius Mites (Acari: Prostigmata: Camerobiidae) for the Turkish Fauna}

\begin{abstract}
In this study, Neophyllobius lamimani McGregor, 1950 and Neophyllobius parthenocissi Bolland 1991, determined in coastal Aegean, identified as new records for the Turkish fauna were redescribed. Mites were taken from soil and litter samples from beneath of Pinus brutia and Thymus sp. and extracted using Berlese funnels and mounted on slides in a modified Hoyer's medium. Then, the specimens were measured and illustrated using a drawing tube attached microscope. Similarities and differences were discussed by comparing them with the other examples of the species spread throughout the world.
\end{abstract}

\section{Article History}

Received: 06.04.2017

Accepted: 27.04.2017

Keywords
Acari,
Camerobiidae,
Neophyllobius,
New record,
Turkey

Research Article

To Cited :Koçyiğit R, Yanar M, Aydın R, Diler A, Güler O 2018. Türkiye Faunası İçin İki Yeni Neophyllobius Akar (Acari: Prostigmata: Camerobiidae) Kaydı. KSÜ Tarim ve Doğa Derg 21(2):106-110, DOI:10.18016/ksudobil.304398.

\section{GiRiş}

Camerobiidae, Raphignathoidea üst familyasının ikinci büyük familyasıdır. Raphignathoidea Grandjean, 1944 (Acari: Prostigmata) üst familyası; Barbutiidae, Caligonellidae, Camerobiidae, Cryptognathidae, Dasythyreidae, Eupalopsellidae, Homocaligidae, Mecognathidae, Raphignathidae, Stigmaeidae ve Xenocaligonellididae familyaları olmak üzere toplam 11 familya, 62 cins ve 900'e yakın türle temsil edilmektedir (Fan ve Zhang 2005, Zhang ve ark., 2011). Türkiye'den ise şuana kadar kaydedilmiş 7 familya, 23 cins ile 175 türü bilinmektedir (Akyol ve ark., 2016).

Camerobiidae, familyasina ait 7 cins bulunur ve bunlardan Neophyllobius Berlese cinsi en fazla türle temsil edilmektedir. Neophyllobius cinsi 1886 yllında Berlese tarafindan $N$. elegans tip türü üzerinden tanımlanmıştır. Neophyllobius cinsine ait türlerin bir çoğu predatör bireyler olarak bilinmekte ve özellikle böcek larvaları ve çeşitli bitki zararlısı akarlarla beslenmektedir (Meyer, 1962; Gerson, 1971, 1973; Gerson ve Smiley, 1990; Gerson ve ark., 1990; Du Toit ve ark., 1998; Bolland, 1991; Bolland ve Mehrnejad, 2001; Khanjani ve Ueckermann, 2002).

Türkiye'den Neophyllobius cinsine ait şimdiye kadar 21 tür tespit edilmiştir. Neophyllobius afyonensis Akyol ve Koç, $N$. askalensis Doğan ve Ayyıldız, $N$. atriplicis Bolland, N. ayvalikensis Akyol, N. ayyildizi Koç ve Madanlar, $N$. bolvadinensis Akyol ve Koç, $N$. communis Bolland, $N$. demirsoyi Akyol ve Koç, $N$. fani Doğan ve Ayyıldız, $N$. izmirensis Akyol, $N$. karabagiensis Akyol ve Koç, N. lachishensis Bolland, $N$. olurensis Doğan ve Ayyıldız, $N$. orhani Doğan ve Ayyıldız, N. persiaensis Khanjani ve Ueckermann, 
2002, N. podocarpi Bolland, N. populous Akyol ve Koç, $N$. quercus Uluçay ve Koç, N. sultanensis Akyol ve Koç, N. turcicus Koç ve Ayyıldız, N. yunusi Akyol ve Koç (Koç ve Ayyıldız, 1996; Koç, 1999, 2001; Koç ve Madanlar, 2002; Doğan ve Ayyıldız, 2003; Akyol ve Koç, 2006 a,b,c; Akyol, 2013; Uluçay ve Koç, 2014; Çobanoğlu ve Yeşilayer, 2016). Bu çalışmayla Neophyllobius cinsine ait Türkiye faunasına iki yeni tür kaydı eklenmiştir.

\section{MATERYAL ve METOT}

Çalışma alanı olarak seçilen Kıyı Ege Bölgesinin, rafignatoid akar faunasinı tespit etmek amaciyla Haziran 2007-Haziran 2008 tarihleri arasinda karasal ve yarı sucul habitatlardan toprak ve döküntü örnekleri alındı. Alınan örneklerin her biri ayrı ayrı naylon torbalar içerisine konulduktan sonra etiketlenip laboratuvara getirildi. Burada her örnek toprak akarları ayıklama düzeneği olan Berlese hunilerine konularak bir hafta süreyle ışık altında bekletildi. Huninin alt kısmında bulunan ve içinde \% 70'lik alkol bulunan toplama şişelerinde biriken akarlar, diseksiyon mikroskobu (stereo mikroskop) altında topraktan ayıklanıp içerisinde laktofenol (laktik asit $50 \mathrm{ml}$, fenol $25 \mathrm{ml}$, saf su $25 \mathrm{ml}$ ) bulunan petri kaplarına ağartılması için bırakıldı. Ağaran akarların modifiye Hoyer ortamında (saf su $50 \mathrm{ml}$, gum arabic $50 \mathrm{gr}$, kloral hidrat $125 \mathrm{gr}$, gliserin $30 \mathrm{ml}$ ) preparatları yapıldı (Koç, 1999, 2001; Koç ve Madanlar, 2002; Doğan ve Ayyıldız, 2003; Akyol ve Koç, 2006 a,b,c). Preparatları yapılan örneklerin çizim takımı bulunan çizim mikroskobunda vücut ve çeşitli organlarının şekilleri çizildi (Şekil 1 ve 2). Şekilleri çizilen örneklerden çeşitli organlarının ölçümleri yapıldı ve ilgili literatür kullanılarak (Bolland, 1991; Bolland ve Mehrnejad, 2001; Khanjani ve Ueckermann, 2002; Doğan ve Ayyıldız, 2003; Akyol ve Koç, 2006 a,b,c; Fan ve Zhang 2005; Khanjani ve ark., 2010; Akyol, 2013) tür teşhisleri yapıldı. 2007- 2008 tarihleri arasında yapılan örneklemelerden elde edilen yeni türler ( $N$. ayvalikensis Akyol ve $N$. izmirensis Akyol) öncelikle verildi (Akyol, 2013). Araştırma alanında yeni kayıtların yeni tür olma ihtimali göz önüne alınarak örneklemelere periyodik olmasa da ara ara devam edildi. Fakat, yeni kayıtlara ait örneklere şuana kadar rastlanmadığı için bu çalışmada verilmiştir.

Sırt ve bacak kıllarının çizim ve isimlendirilmesi Kethley (1990) ve Grandjean (1944)'a göre yapılmıştır. Bütün ölçümler mikrometre $(\mu \mathrm{m})$ olarak verilmiştir. Tip örneği ve diğer örnekler Manisa Celal Bayar Üniversitesi, Fen-Edebiyat Fakültesi, Biyoloji Bölümü, Zooloji laboratuarında, preparat halinde saklanmaktadır.

\section{BULGULAR}

Familya: Camerobiidae Southcott, 1957
Cins: Neophyllobius Berlese, 1886

Tip Türü: Neophyllobius elegans Berlese, 1886.

Dişi: İdiozoma 14-15 çift kıl taşır. Sırt kılları genellikle uzun, kılıç gibi ve üzerleri tüberküllüdür. Peritremler gnatozomanın üst kısmında yer alır ve genellikle birleşik lobludur. Femur 2 kıl, genu 1 kıl, tibia $3 \mathrm{kıl}$ ve 1 tane kılıç şeklinde kıl ve muhtemelen iz halinde tibial tırnak taşır. Tarsusta 2 kıl, 1 solenidium ve 1 veya 2 eupatidium bulunur. Bacakları oldukça uzundur; tarsus I ve II'nin arka kısmında birer tane solenidium yer alır. Bazı türlerin tarsus IV'ü bir tane orta konumlu kıllı iken, birçok türün tarsusları iki tane orta konumlu kıl taşır. Tibiaların kıl sayısı 9-88-7 şeklindedir.

Genital kapakta 1 çift genital kıl, anal kapakta 3 çift pseudanal kıl bulunur.

Erkek: Tibia I' de fazladan bir solenidium vardır. Tarsus I-IV' ün her biri genişlemiş bir solenidium taşır.

Tür: Neophyllobius lamimani McGregor, 1950 (Şekil 1) Dişi: Vücut, gnatozoma hariç $322 \mu \mathrm{m}$ uzunluğunda ve $280 \mu \mathrm{m}$ genişliğindedir

Gnatozoma $62 \mu \mathrm{m}$ uzunluğundadır. Subkapitulumun orta kısmında bir çift düz kıl $(m=13 \mu \mathrm{m})$ vardır. Palp trokanterinde kll yoktur. Femurda 2 , genuda 1 , tibiada $3+1$, tarsusda ise $2 \mathrm{kl}, 1$ solenidium ve 1 eupatidium vardır (Şekil 1. G).

Sırtta toplam 15 çift dişli ve kalın kıl vardır. Bunlardan 6 çifti merkezi kıl olup idiozomanın orta kısmına yerleşmiştir. Sırt kılları küçük dişlidir. İki çift göz sci ve sce kılları arasında yer alır. Sırt tarafı kaba çizgilidir. Çizgiler $f_{2}$ kılının kaidesine kadar ve vücudun kenar kısmında boyuna, merkezi kıllar arasında ise enine olarak uzanır. Sirt kıllarının uzunlukları şu şekildedir: $v i=70 \mu \mathrm{m}, v e=44 \mu \mathrm{m}, s c \dot{=}$ $52 \mu \mathrm{m}, s c e=55 \mu \mathrm{m}, c_{1}=42 \mu \mathrm{m}, c_{2}=81 \mu \mathrm{m}, d_{1}=52 \mu \mathrm{m}$, $d_{2}=52 \mu \mathrm{m}, e_{1}=83 \mu \mathrm{m}, e_{2}=55 \mu \mathrm{m}, f_{1}=68 \mu \mathrm{m}, f_{2}=31 \mu \mathrm{m}$, $h_{1}=31 \mu \mathrm{m}, h_{2}=29 \mu \mathrm{m}, p d x=45 \mu \mathrm{m}$ (Şekil 1. A).

Karın tarafinda birinci koksa üzerinde, üçüncü koksalar arasında ve genital bölgenin üst kısmında olmak üzere üç çift düz kıl $(1 a=21 \mu \mathrm{m}, 3 a=26 \mu \mathrm{m}, 4 a=$ $18 \mu \mathrm{m})$ vardır. Bacak koksaları (I+II ve III+IV) şeklinde olmak üzere iki grup halindedir ve karın bölgesinin diğer bütün tarafı çizgilidir. Aggenital bölgede bir çift aggenital kll (ag), genital kapak üzerinde bir çift genital kıl $(g)$ ve anal kapak üzerinde 3 çift pseudanal kıl ( $\left.p_{S_{1-3}}\right)$ bulunur (Şekil 1. B).

Bacakları idiozomadan uzundur. Bacakların uzunlukları (femurun kaidesinden tarsus tırnaklarının ucuna kadar) şu şekildedir: I. bacak $500 \mu \mathrm{m}$, II. bacak $416 \mu \mathrm{m}$, III. bacak $468 \mu \mathrm{m}$ ve IV. bacak $468 \mu \mathrm{m}$. Birinci bacaktan dördüncü bacağa kadar kılların bacak parçalarına dağılımı (solenidiumların sayısı parantez içerisinde gösterilmiştir) şöyledir: koksa 3-1-2-2, trokanter 1-1$1-1$, femur $4-3-2-2$, genu $1(+k)-1(+k)-1-1$, tibia $9(+\varphi)$ 
$8(+\varphi)-8(+\varphi)-7(+\varphi)$, tarsus $10(+\omega)-10(+\omega)-8-7$. Tarsus I ve IV' de iki tane orta konumlu kıl bulunur (Şekil 1. C-F). Erkek: Bilinmiyor.

İncelenen Örnek ve Yaşam Alanı: Balıkesir İli, Edremit İlçesi, Kazdağları, Edremit Körfezi, 50 m, 24. 05.2008, kızılçam (Pinus brutia) altı döküntü ve toprak örneği, 1 ㅇ. Yayılışı: Amerika Birleşik Devletleri (A.B.D.) (California, 1936, E.W.Baker), Türkiye (Balıkesir, Edremit Körfezi, 2008, M. Akyol).

Tür: Neophyllobius parthenocissi Bolland, 1991 (Şekil 2) Dişi: Vücut, gnatozoma hariç (iki örneğe ait ölçüm) 291$302 \mu \mathrm{m}$ uzunluğunda ve 239-250 $\mu \mathrm{m}$ genişliğindedir.

Gnatozoma $62 \mu \mathrm{m}$ uzunluğundadır. Subkapitulumun orta kısmında bir çift düz kıl $(m=13 \mu \mathrm{m})$ vardır. Palp trokanterinde kll yoktur. Femurda 2, genuda 1, tibiada $3+1$, tarsusda ise $2 \mathrm{kl}, 1$ solenidium ve 1 eupatidium vardır (Şekil 2. G).

Surtta toplam 15 çift dişli ve kalın kıl vardır. Bunlardan
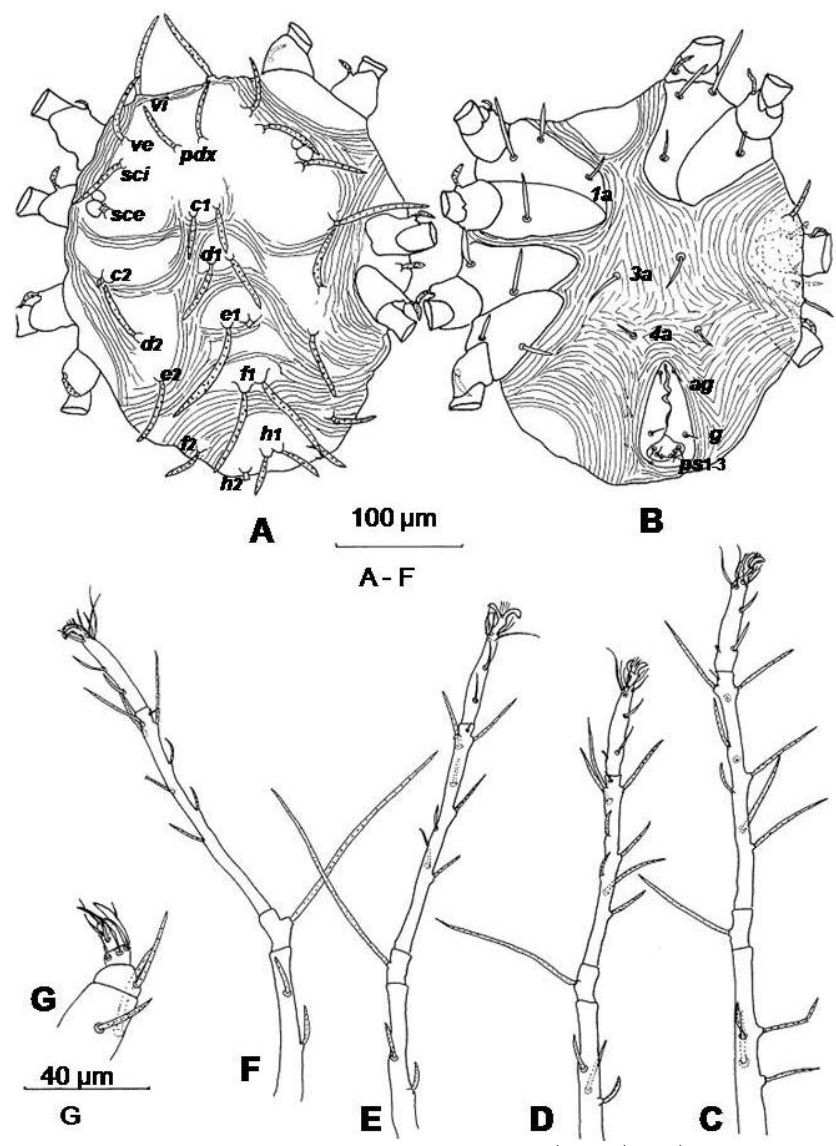

Şekil 1. Neophyllobius lamimani (Dişi). A) Vücut, üstten, B) Vücut, alttan, C) I. bacak, D) II. bacak, E) III. bacak, F) IV. bacak, G) Palp
6 çifti merkezi kıl olup idiozomanın orta kısmına yerleşmiştir. Sırt kılları küçük dişlidir. İki çift göz sci ve sce kılları arasında yer alır. Sırt tarafi kaba çizgilidir. Cizgiler f2 kılının kaidesine kadar ve vücudun kenar kısminda boyuna, merkezi kıllar arasında ise enine olarak uzanır. Sırt kıllarının uzunlukları şu şekildedir: vi $=73 \mu \mathrm{m}, v e=62 \mu \mathrm{m}, s c i=55 \mu \mathrm{m}, s c e=57-60 \mu \mathrm{m}, c 1=$ $112 \mu \mathrm{m}, c 2=96 \mu \mathrm{m}, d 1=125 \mu \mathrm{m}, d 2=65 \mu \mathrm{m}, e 1=112-117$ $\mu \mathrm{m}, e 2=68 \mu \mathrm{m}, f 1=91 \mu \mathrm{m}, f 2=39 \mu \mathrm{m}, h 1=42-44 \mu \mathrm{m}, h 2=$ 34-36 $\mu \mathrm{m}, p d x=60-65 \mu \mathrm{m}$ (Şekil 2. A).

Karın tarafında birinci koksa üzerinde, üçüncü koksalar arasında ve genital bölgenin üst kısmında olmak üzere üç çift düz kıl ( $1 a=21 \mu \mathrm{m}, 3 a=26 \mu \mathrm{m}, 4 a=13 \mu \mathrm{m})$ vardır. Bacak koksaları (I+II ve III+IV) şeklinde olmak üzere iki grup halindedir ve karın bölgesinin diğer bütün tarafi çizgilidir. Aggenital bölgede bir çift aggenital kıl (ag), genital kapak üzerinde bir çift genital kıl $(g)$ ve anal kapak üzerinde 3 çift pseudanal kıl (ps1-3) bulunur (Şekil 2. B).

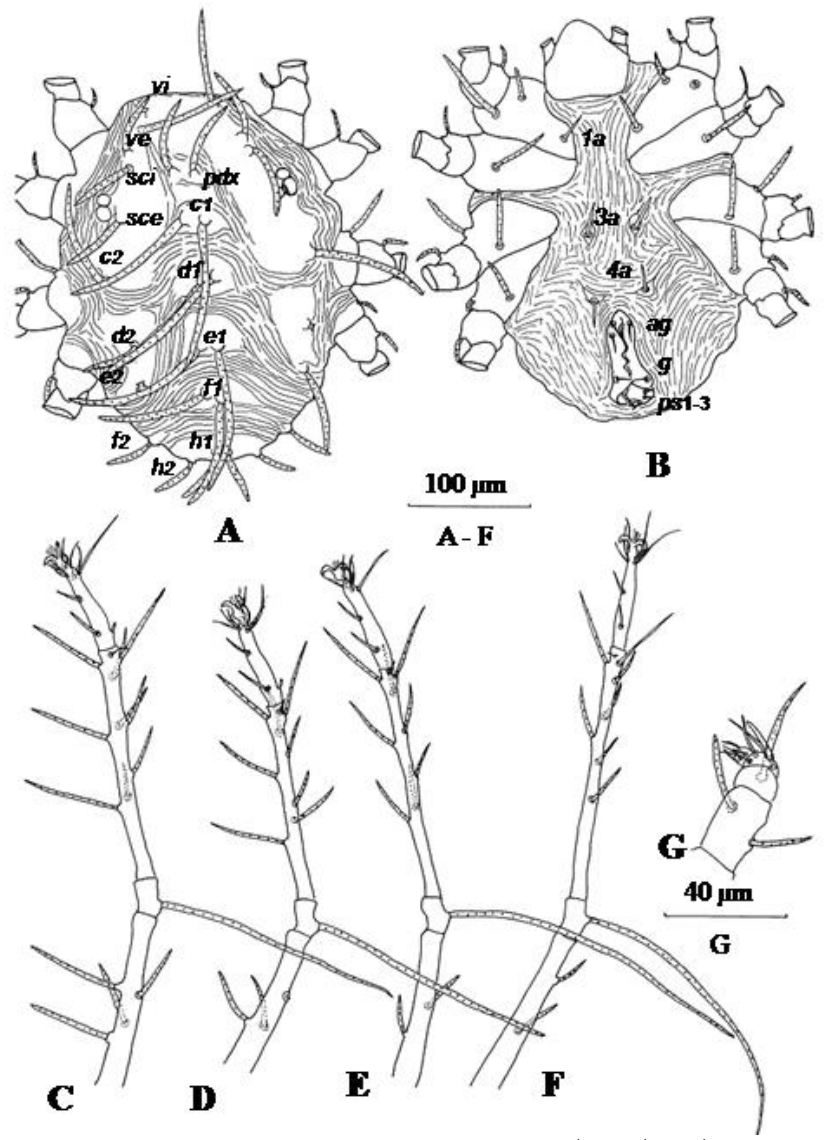

Şekil 2. Neophyllobius parthenocissi (Dişi). A) Vücut, üstten, B) Vücut, alttan, C) I. bacak, D) II. bacak, E) III. bacak, F) IV. bacak, G) Palp. 
Bacakları idiozomadan uzundur. Bacakların uzunlukları (femurun kaidesinden tarsus tırnaklarının ucuna kadar) şu şekildedir: I. bacak 437$468 \mu \mathrm{m}$, II. bacak $416 \mu \mathrm{m}$, III. bacak $437-458 \mu \mathrm{m}$ ve IV. bacak $468 \mu \mathrm{m}$. Birinci bacaktan dördüncü bacağa kadar kılların bacak parçalarına dağılımı (solenidiumların sayısı parantez içerisinde gösterilmiştir) şöyledir: koksa 3-1-2-2, trokanter 1$1-1-1$, femur $4-3-2-2$, genu $1(+k)-1(+k)-1-1$, tibia $9(+\varphi)-8(+\varphi)-8(+\varphi)-7(+\varphi)$, tarsus $10(+\omega)-10(+\omega)-8-7$. Tarsus I ve IV'de iki tane orta konumlu kıl bulunur (Şekil 2. C-F).

Erkek: Dişiye göre küçük vücutlu, sırttaki $h 1$ kılı oldukça kısalmış, tibia I’in uç kısmında iki solenidia bulunur.

İncelenen Örnekler ve Yaşama Alanları: İzmir İli, Karaburun İlçesi, Sarpıncık köyü, 260 m, 16.03.2008, kekik (Thymus sp.) altı döküntü ve toprak örneği, 1우우 anormal.

Yayılışı: Amerika Birleşik Devletleri (Washington, 1950, E.W.Baker), Türkiye (İzmir, Karaburun, 2008, M. Akyol).

\section{TARTIŞMA ve SONUÇ}

Neophyllobius lamimani McGregor, 1950 A.B.D (California)'de ağaç üzeri liken, asma ve tuzcul çimen örneğinden bulunan dişi üzerinden McGregor tarafindan tanımlanmıştır (Bolland, 1991). Bu çalışmadaki örneği ise Kıyı Ege'de yer alan Balıkesir ili Edremit Körfezi kızılçam (Pinus brutia) altından alınan döküntü ve toprak örneğinde bulunmuştur.

A.B.D (California) örneğinde vücut büyüklüğü 405/330 $\mu \mathrm{m}$ verilmiştir (Bolland, 1991). Türkiye örneğinin vücut büyüklüğü $322 / 280 \mu \mathrm{m}$ olarak bulunmuştur. Buna göre, Türkiye örneği vücut büyüklüğü bakımından A.B.D (California) örneğinden küçük olduğu anlaşılmaktadır. A.B.D (California) örneğinde merkezi sırt killarının uzunlukları: $p d x=90 / 85 \mu \mathrm{m}, c_{1}=$ $85 \mu \mathrm{m}, d_{1}=80 \mu \mathrm{m}, e_{1}=75 \mu \mathrm{m}, f_{1}=70 \mu \mathrm{m}, h_{1}=30 \mu \mathrm{m}$, yanal sirt kıllarının uzunlukları: $v \dot{i}=70 \mu \mathrm{m}, v e=55$ $\mu \mathrm{m}, s c i=70 \mu \mathrm{m}, s c e=60 \mu \mathrm{m}, c_{2}=80 \mu \mathrm{m}, d_{2}=60 \mu \mathrm{m}, e_{2}=$ $55 \mu \mathrm{m}, f_{2}=50 \mu \mathrm{m}, \quad h_{2}=35 \mu \mathrm{m}$ olarak bulunmuştur (Bolland, 1991). Türkiye örneğinin merkezi sırt kıllarınin uzunluklar1: $p d x=45 \mu \mathrm{m}, c_{1}=42 \mu \mathrm{m}, d_{1}=52$ $\mu \mathrm{m}, e_{1}=83 \mu \mathrm{m}, f_{1}=68 \mu \mathrm{m}, h_{1}=31 \mu \mathrm{m}$, yanal sirt killarmin uzunluklarl: $v i=70 \mu \mathrm{m}, v e=44 \mu \mathrm{m}, s c i=52$ $\mu \mathrm{m}, s c e=55 \mu \mathrm{m}, c_{2}=81 \mu \mathrm{m}, d_{2}=52 \mu \mathrm{m}, e_{2}=55 \mu \mathrm{m}, f_{2}=$ $31 \mu \mathrm{m}, h_{2}=29 \mu \mathrm{m}$ olarak tespit edilmiştir. A.B.D (California) örneğinde merkezi sırt kıllarından $p d x, c 1$, ve $d_{1}$ kıllarının Türkiye örneğinden neredeyse iki katı kadar uzun olduğu görülmektedir. Türkiye örneği diğer yapısal özellikleri bakımından tip örneğine benzerlik göstermektedir.

Neophyllobius parthenocissi Bolland, 1991 A.B.D (Washington)'de Parthenocissus tricuspidata bitkisi üzerinden bulunan dişi üzerinden Bolland tarafından tanımlanmıştır (Bolland, 1991). Türkiye örneği, İzmir ili Karaburun Yarımadası kekik (Thymus sp.) altından alınan döküntü ve toprak örneğinde bulunmuştur.

A.B.D (Washington) örneğinde vücut büyüklüğü 310/275 $\mu \mathrm{m}$ verilmiştir (Bolland, 1991). Türkiye örneğinin vücut büyüklüğü 291-302/239-250 $\mu \mathrm{m}$ olarak bulunmuştur. Buna göre, Türkiye örneği vücut büyüklüğü bakımından A.B.D (Washington) örneği ile yaklaşık aynı büyüklüktedir. A.B.D (California) örneğinde merkezi sırt kıllarının uzunlukları: $p d x=85$ $\mu \mathrm{m}, c_{1}=125 \mu \mathrm{m}, d_{1}=120 \mu \mathrm{m}, e_{1}=125 \mu \mathrm{m}, f_{1}=85 \mu \mathrm{m}$, $h_{1}=45 \mu \mathrm{m}$, yanal sirt kıllarının uzunlukları: $v i=65$ $\mu \mathrm{m}, v e=75 \mu \mathrm{m}, s c \dot{=}=80 \mu \mathrm{m}, s c e=70 \mu \mathrm{m}, c_{2}=85 \mu \mathrm{m}, d_{2}=$ $70 \mu \mathrm{m}, e_{2}=75 \mu \mathrm{m}, f_{2}=50 \mu \mathrm{m}, \quad h_{2}=45 \mu \mathrm{m}$ olarak bulunmuştur (Bolland, 1991). Türkiye örneğinin merkezi sırt kıllarının uzunlukları: $p d x=60-65 \mu \mathrm{m}$, $c_{1}=112 \mu \mathrm{m}, d_{1}=125 \mu \mathrm{m}, e_{1}=112-117 \mu \mathrm{m}, f_{1}=91 \mu \mathrm{m}$, $h_{1}=42-44 \mu \mathrm{m}$, yanal sirt kıllarinın uzunlukları: $v \dot{=}=$ $73 \mu \mathrm{m}, v e=62 \mu \mathrm{m}, s c \dot{=}=55 \mu \mathrm{m}, s c e=57-60 \mu \mathrm{m}, c_{2}=96$ $\mu \mathrm{m}, d_{2}=65 \mu \mathrm{m}, e_{2}=68 \mu \mathrm{m}, f_{2}=39 \mu \mathrm{m}, h_{2}=34-36 \mu \mathrm{m}$ olarak tespit edilmiştir. A.B.D (Washington) örneğinde sırt kıllarından $p d x$ ve sci kıllarının Türkiye örneğinden uzun olduğu görülmektedir. Türkiye örneği diğer yapısal özellikleri bakımından tip örneğine benzerlik göstermektedir.

\section{KAYNAKLAR}

Akyol M, Uluçay İ, Koç K 2016. Symposium on Euro Asian Biodiversity, 23-27 Mayıs 2016, Antalya, Turkey.

Akyol M 2013. Two new species of the genus Neophyllobius Berlese (Acari: Camerobiidae) from Turkey, International Journal of Acarology, 39(7): 542-546.

Akyol M, Koç K 2006a. The camerobiid mites (Acari, Camerobiidae) of Turkey. Biologia, Bratislava, 61(2): 125-132.

Akyol M, Koç K 2006b. Two new species of Neophyllobius (Acari: Camerobiidae) from Turkey. Zootaxa, 1196: 63-68.

Akyol M, Koç K 2006c. New species of Neophyllobius and Tycherobius (Acari: Camerobiidae) from Turkey. Biologia Bratislava, 61: 487-495.

Berlese A 1886. Acari dannosi alle piante coltivate. Padova: $31 \mathrm{pp}$

Bolland HR 1991. Review of the systematics of the family Camerobiidae, II. The genus Neophyllobius Berlese, 1886 (Acari: Raphignathoidea). Genus, 2 (2): 59-226.

Bolland HR, Mehrnejad MR 2001. Neophyllobius pistaciae Bolland and Mehrnejad (Acari: Camerobiidae) from Iran. International Journal of Acarology, 27(1): 49-53.

Çobanoğlu S, Yeşilayer A 2016. Neophyllobius persiaensis Khanjani ve Ueckermann (Acari: Camerobiidae); new record of the species from the parks and ornamental plants in İstanbul (Turkey). Türkiye Entomoloji Bülteni, 6(1): 3-8. 
Doğan S, Ayylldız N 2003. New species of Neophyllobius (Acari: Camerobiidae) and description of Cryptognathus ozkani (Acari: Cryptognathidae) male from Turkey. Biologia, 58(2): 121-132.

Du Toit BJ, Theron, PD, Ueckermann EA 1998. A new genus and four new species of the family Camerobiidae (Acari: Raphignathoidea) from South Africa. International Journal of Acarology, 24(1): 3 -19 .

Fan Q-H, Zhang Z-Q 2005. Raphignathoidea (Acari: Prostigmata). Fauna of New Zealand, 52: 400.

Gerson U 1971. The mites associated with citrus in Israel. Israel Journal Entomology, 6: 5-21.

Gerson U 1973. The mites associated with armored scale insects. Proc. 3rd Int. Congr. Acarol. Prague, 1971: 653-654.

Gerson U, Oconnor BM, Houck MA 1990. 2. 2. 6 Acari, pp. 77- 97. In: Rosen, D. (ed.).The Armored scale insects. Their biology, natural enemies and control, Vol. 4B. Elsevier Publishers, Amsterdam.

Gerson U, Smiley RL 1990. Acarina Biocontrol Agents. Chapman and Hall, London, 96-97.

Grandjean F, 1944. Observations sur les acariens de la famille des Stigmaeidae. Archives des Sciences physiques et naturelles, 26: 103-131.

Kethley J 1990. Acarina: Prostigmata (Actinedida). In Soil Biology Guide, ed. D.L. Dindal.- John Wiley and Sons, New York, 667-756.

Khanjani M, Ueckermann EA 2002. Camerobiidae of Iran with descriptions of three new species (Acari: Camerobiidae). Systematic and Applied Acarology. Systematic and Applied Acarology Society, 7: 159166.
Khanjani M, Fayaz BA, Ghanbalani GN 2010. Two new species of the genus Neophyllobius Berlese (Acari: Camerobiidae) from Iran. Zootaxa. 2521:5364.

Koç K 1999. Neophyllobius communis and its developmental stages (Acari: Camerobiidae). Entomologische Berichten, Amsterdam, 59(8): 119123.

Koç K 2001. A new record of Neophyllobius Berlese (Acari: Camerobiidae) for the fauna of Turkey. Türkiye Entomoloji Dergisi, 25(4): 257-262.

Koç K, Ayyıldız N 1996. A new species of the Neophyllobius (Acari, Camerobiidae) from Turkey. International Journal of Acarolgia, 22 (4): 291 294.

Koç K, Madanlar N 2002. A new species of Neophyllobius Berlese (Acari: Camerobiidae) from Turkey. Acarologia, 1: 61-66.

Meyer MKP 1962. Two new predators of red scale (Aonidiella auranti) in South Africa. South African Journal of Agricultural Science, 5(3): 411- 417.

Uluçay İ, Koç K 2014. A new species of Neophyllobius and description of male of Neophyllobius yunusi (Acari: Camerobiidae) from Turkey. International Journal of Acarology, 40(1), 15-22.

Zhang Z-Q, Fan Q-H, Pesic V, Smit H, Bochkov AV, Khaustov AA, Baker A, Wohltmann A, Wen T-H, Amrine JW, Beron P, Lin J-Z, Gabrys G, Husband R 2011. Order Trombidiformes Reuter 1909. In: Zhang, Z-Q. (ed.) Animal biodiversity: an outline of higher-level classification and survey of taxonomic richness. Zootaxa, 3148: 129-138. 